\title{
Differential Contribution of Substance P and Neurokinin A to Spinal Cord Neurokinin-1 Receptor Signaling in the Rat
}

\author{
Jodie A. Trafton, Catherine Abbadie, and Allan I. Basbaum \\ Departments of Anatomy and Physiology and W. M. Keck Foundation for Integrative Neuroscience, University of \\ California San Francisco, San Francisco, California 94143
}

Although the tachykinins substance $P(S P)$ and neurokinin $A$ (NKA) are coreleased from primary afferent nociceptors and act via neurokinin (NK) receptors, their differential effects in vivo are not known. Despite pharmacological evidence that NKA preferentially binds NK-2 receptors, this receptor is not found in spinal cord neurons. Thus, in the present studies, we compared the extent to which SP and NKA contribute to spinal nociceptive processing via the $\mathrm{NK}-1$ receptor.

We found that SP and NKA induce NK-1 receptor internalization with identical dose dependence and induce increases in intracellular calcium at the same concentrations, suggesting that SP and NKA equally activate the NK-1 receptor. We found, however, that the selective $\mathrm{NK}-1$ receptor antagonist GR 205171 blocked NKA but not SP-induced NK-1 receptor internalization in the rat spinal cord in vivo and in embryonic day 19 rat spinal neurons in vitro. Using this selectivity of GR 205171

The tachykinins substance $\mathrm{P}$ (SP) and neurokinin A (NKA) are produced from a single precursor, the preprotachykinin A $(p p t-A)$ gene, by a population of small-diameter primary afferent neurons. SP and NKA are costored in dense core vesicles and can be released in response to noxious stimulation. There is electrophysiological evidence that both SP and NKA can activate dorsal horn neurons (Henry, 1976; De Koninck and Henry, 1991; Radhakrishnan and Henry, 1997) and induce pain behaviors after intrathecal injection (Hylden and Wilcox, 1981; Gamse and Saria, 1986). Despite these comparable effects in the spinal cord, it is not clear whether the actions of one tachykinin completely mimics the other, (i.e., whether there is redundancy of these PPT-A products) or whether different receptors, located on different populations of neurons, mediate the actions of these peptides.

The tachykinins target three neurokinin receptors (NK-1, NK-2, and NK-3). Early binding studies indicated that each of the

\footnotetext{
Received Aug. 28, 2000; revised March 5, 2001; accepted March 5, 2001.

This research was supported by National Institute of Health Grants NS 14627, NS 21445, and DE 08973. J.A.T. was supported in part by a National Science Foundation predoctoral fellowship, an Achievement Rewards for College Scientists scholarship, and the Veterans Affairs Health Services Research and Development Service. C.A. was supported by Institut National de la Santé et de la Recherche Médicale and Institut UPSA de la douleur.

Correspondence should be addressed to Allan I. Basbaum, 513 Parnassus Avenue, Box 0452, San Francisco, CA 94143-0452. E-mail: aib@phy.ucsf.edu.

J. A. Trafton's present address: Veterans Affairs Health Services Research and Development Service Center for Health Care Evaluation, 795 Willow Road (152 MPD), Menlo Park, CA 94025

C. Abbadie's present address: Laboratory of Molecular Neuropharmacology, Memorial Sloan-Kettering Cancer Center, New York, NY 10021.

Copyright (C) 2001 Society for Neuroscience $0270-6474 / 01 / 213656-09 \$ 15.00 / 0$
}

for NKA-induced NK-1 receptor activation, we examined the relative contribution of $\mathrm{SP}$ and NKA to noxious stimulusinduced activation of spinal NK-1 receptors. We estimate that NKA contributes to at least $50 \%$ of the $\mathrm{NK}-1$ receptor activation in lamina I. Under inflammatory conditions, all noxious stimulus-induced NK-1 receptor internalization in deep dorsal horn neurons was blocked by GR 205171, suggesting that it is entirely NKA-mediated. Substance P-mediated NK-1 receptor internalization was focused at the site of termination of stimulated nociceptors but NKA also activated NK-1 receptors at more distant sites. We conclude that NKA not only targets the NK-1 receptor but may be a predominant pronociceptive primary afferent neurotransmitter.

Key words: rat; tachykinin; dorsal horn; nociception; internalization; inflammation cloned receptors has a higher affinity for a different tachykinin. This led to the proposal that SP, NKA, and NKB are endogenous ligands for the NK-1, NK-2, and NK-3 receptors, respectively. Binding and pharmacological studies using selective antagonists have provided support for this categorization. For example, the selective NK-2 receptor antagonist SR 48968 blocks C fiberinduced long-term potentiation in the spinal cord, reduces the responses of dorsal horn neurons to mechanical stimulation and the hyperexcitability of these responses after inflammatory injury, and reduces the hyperalgesic effects of intrathecal NKA in the tail flick test (Neugebauer et al., 1996; Yashpal et al., 1996; Liu and Sandkühler, 1997). However, there is no evidence of NK-2 receptor expression in the CNS, except in a limited glial population (Zerari et al., 1998). As a result, it is difficult to account for the apparent NK-2-selective antagonism in the CNS, and thus the target through which NKA exerts effects in the CNS is unclear.

The paradox may be partly explained by a growing literature that suggests that the affinity of NKA for the NK-1 receptor is actually comparable with that of SP. Indeed, Hastrup and Schwartz (1996), using homologous rather than heterologous binding studies, found that the affinity of NKA for the NK-1 receptor was much higher than measured previously. Furthermore, both SP and NKA increase $\mathrm{IP}_{3}$ production and induce inward currents via the NK-1 receptor at similar concentrations (Akasu et al., 1996; Sagan et al., 1996). These findings raise the possibility that NKA is an important NK-1 receptor ligand in vivo. Consistent with this view, electrophysiological studies demonstrate that exogenously applied NKA activates dorsal horn, wide dynamic range neurons via the NK-1 receptor (Radhakrish- 
nan and Henry, 1997). To date, however, no studies have examined the extent to which effects of endogenously released NKA are mediated via the $\mathrm{NK}-1$ receptor.

Here, we directly assess the NK-1 receptor-mediated effects of $\mathrm{SP}$ and NKA in vivo and in vitro. As a functional marker of NK-1 receptor activation, we monitor $\mathrm{NK}-1$ receptor internalization, which we have shown previously occurs in response to substance $\mathrm{P}$ and correlates quantitatively with $\mathrm{NK}-1$ receptor-mediated increases in intracellular calcium (Trafton et al., 1999). Additionally, we provide evidence that the NK-1 receptor antagonist GR 205171 profoundly inhibits NKA compared with SP-mediated NK-1 receptor activation. Next, using GR 205171 to selectively block NKA-induced NK-1 receptor activation, we examined the contribution of NKA to noxious stimulus-induced NK-1 receptor activation in the spinal cord.

\section{MATERIALS AND METHODS}

All experiments were reviewed and approved by the Institutional Care and Animal Use Committee at the University of California, San Francisco (UCSF).

\section{Cell culture experiments}

Spinal cord cultures. Spinal cord cultures were prepared from embryonic day 19 Sprague Dawley rats using a modification of the method of Yu et al. (1984). Spinal cords were dissected out, washed, and treated with a standard $0.25 \%$ trypsin-versene mixture (STV) (cell culture facility, UCSF) for $12 \mathrm{~min}$. The cords were again washed and mechanically dissociated with a large bore pipette. Cells from each cord were diluted into $24 \mathrm{ml}$ of MEM-PAK buffer (cell culture facility, UCSF) supplemented to contain 5\% normal horse serum, $5 \%$ fetal calf serum, and $1 \times$ penicillin-streptomycin, $30 \mathrm{~mm}$ glucose, and $2 \mathrm{~mm}$ glycine. Cells were plated on glass coverslips (Carolina Biological Supply Company, Burlington, NC) or eight-well cover glass (Fisher, Santa Clara, CA) and incubated at $37^{\circ} \mathrm{C}$ in a humidified incubator with $5 \% \quad \mathrm{CO}_{2}-95 \% \mathrm{O}_{2}$. Cultures were used at 5-6 d of age.

Calcium imaging. Spinal cord cultures plated on eight-well cover glass were incubated in CI buffer (in mM: $130 \mathrm{NaCl}, 0.3 \mathrm{KCl}, 2.5 \mathrm{CaCl} 2,0.6$ $\mathrm{MgCl} 2,1.2 \mathrm{NaHCO}, 10$ glucose, and 10 HEPES, pH 7.4) containing 10 $\mu \mathrm{M}$ fura-2 AM and $0.02 \%$ pleuronic acid (Molecular Probes, Eugene, OR) for $25 \mathrm{~min}$. Fura-2 was then removed and replaced with $150 \mu \mathrm{l} /$ well fresh CI buffer. We performed ratiometric calcium imaging with a Nikon (Tokyo, Japan) Diaphot fluorescence microscope equipped with a variable filter wheel (Sutter Instruments, Novato, CA) and an intensified CCD camera (Hamamatsu, Bridgewater, NJ). Dual images (340 and 380 $\mathrm{nm}$ excitation, $510 \mathrm{~nm}$ emission) were collected every $4 \mathrm{sec}$. For each well, we recorded five baseline images, and then $150 \mu \mathrm{l}$ of SP in CI buffer at twice the desired final concentration was pipetted into the well. Responses were recorded for the following $40 \mathrm{sec}$.

The average 340/380 ratio was calculated for each of the cells in the field and for each of the time points. All cells that showed an average increase in 340/380 ratio greater than twice the average baseline 340/380 ratio for that cell were considered responders. Only responders were considered in the subsequent analysis. The total increase in intracellular calcium was calculated for all responders in each well by taking the sum of the $340 / 380$ ratios over the $40 \mathrm{sec}$ after SP application with the average baseline values measured over the first $20 \mathrm{sec}$ subtracted from those values.

In vitro internalization. Spinal cord cultures plated on coverslips were incubated in culture media containing the indicated concentration of tachykinin. After 15 min, media was removed, and the cells were fixed in $10 \%$ formalin for $20 \mathrm{~min}$. The NK-1 receptor was labeled for immunofluorescent analysis as described below for tissue slices. Confocal images ( $75 \times 4.0$ iris diameter) were taken of five NK-1 receptor-positive neurons per coverslip, and the number of endosomes in each cell was counted and averaged for neurons over the coverslip. Images were collected on an MRC 1024 confocal microscope (Bio-Rad, Hercules, $\mathrm{CA}$ ). The investigator taking images and counting endosomes was unaware of the treatment that the cultures received. All neurons containing $>10$ endosomes were considered to have internalized the NK-1 receptor, and from the endosome counts described above, for each coverslip we calculated the percentage of NK-1 receptor-positive neurons that showed internalized receptor.
Statistical analysis. The percent of maximal observed effect was calculated using the following formula: (average value with tachykinin dose (x) - average value with $0 \mathrm{~mm}$ tachykinin)/highest observed average value with any tachykinin dose.

The percent of maximal observed responders was calculated using the following formula: (\% of responders with tachykinin dose $(\mathrm{x})-\%$ of responders with $0 \mathrm{~mm}$ tachykinin)/highest $\%$ of responders observed with any tachykinin dose.

The threshold values that we chose for counting cells as responders were based on the baseline and maximal response values. Similar results were obtained when we performed the analysis using 15 endosomes and 1.5 times baseline as thresholds. $\mathrm{EC}_{50}$ values were calculated with Prism (GraphPad Software Inc., San Diego, CA). For statistical analysis, we used a two-way ANOVA for measurement of intracellular calcium concentration or NK-1 receptor internalization and for tachykinin dose.

\section{In vivo experiments}

Experimental animals. Experiments were performed on male Sprague Dawley rats (Bantin-Kingman, Fremont, CA), weighing 230-270 gm. Noxious mechanical and thermal stimulation and intrathecal injections were performed $10-15$ min after the rats were anesthetized with sodium pentobarbital $(50 \mathrm{mg} / \mathrm{kg}$, i.p.). This dose blocked flexor reflex responses to hindpaw stimulation. In some rats, inflammation was induced by subcutaneous injection of $100 \mu \mathrm{l}$ of complete Freund's adjuvant (CFA) (killed mycobacterium butyricum suspended in mineral oil; solution at 10 $\mathrm{mg} / \mathrm{ml}$; Sigma, St. Louis, MO) in the left hindpaw. Rats were stimulated $2 \mathrm{~d}$ after the inflammation was induced.

Injections. The NK-1 receptor antagonist GR 205171 (kindly provided by Glaxo-Wellcome, Stevenage, UK) in $1.0 \mathrm{ml}$ of saline was injected subcutaneously in the back of the neck, 20 min before treatment. Intrathecal injections in $20 \mu \mathrm{l}$ of saline were made directly between the S1-S2 vertebrae with a $271 / 2$-gauge needle. Control animals received an equal volume of saline. Placement of the needle in the intrathecal space was verified by a lateral flick of the tail. The NK-2 receptor antagonist MEN-11420 (kindly provided by Carlos Maggi at Menarini Research, Florence, Italy) was injected $10 \mathrm{~min}$ before the mechanical stimulus or the intrathecal NKA injection.

Hindpaw stimulation. Noxious mechanical stimulation (pinch) was applied to the distal part of one hindpaw with a hemostat for $15 \mathrm{sec}$. For thermal stimulation, the rat's hindpaw (to just below the ankle) was dipped for 2 min into a water bath heated to $51^{\circ} \mathrm{C}$. The rats were perfused 5 min after the stimulation ended.

Tissue preparation and immunofluorescent labeling. At the appropriate time, the animals received an additional injection of sodium pentobarbital $(100 \mathrm{mg} / \mathrm{kg}$, i.p.) and were perfused intracardially with $50 \mathrm{ml}$ of PBS $0.1 \mathrm{M}$, followed by $500 \mathrm{ml}$ of $10 \%$ formalin in $0.1 \mathrm{M}$ phosphate buffer (PB). After the perfusion, the lumbar spinal cord was removed, post-fixed for $4 \mathrm{hr}$ in the same fixative, and then cryoprotected overnight in $30 \%$ sucrose in $0.1 \mathrm{M} \mathrm{PB}$. Immunostaining was performed on $30 \mu \mathrm{m}$ lumbar spinal cord sections (from segments L2-L6) cut in the sagittal plane on a freezing microtome. The tissue sections were incubated for $30 \mathrm{~min}$ at room temperature in a blocking solution of $3 \%$ normal goat serum in PBS with $0.3 \%$ Triton X-100 (NGST).

For immunofluorescent staining of the NK-1 receptor, the sections were incubated overnight in the primary antiserum, diluted to 1:5000. The characteristics of the antiserum, directed against the $\mathrm{C}$-terminal tail of the NK-1 receptor, have been described previously (Vigna et al., 1994). After the primary antiserum, the sections were washed three times in $1 \%$ NGST and then incubated in indocarbocyanine Cy3-conjugated goat anti-rabbit IgG (1: 600; Jackson ImmunoResearch, West Grove, PA) for $2 \mathrm{hr}$ at room temperature.

Quantification of internalization. We quantified NK-1 receptor internalization as described previously (Abbadie et al., 1997). Briefly, to analyze internalization in cell bodies, we used a $20 \times$ objective on a Nikon FXA microscope equipped for fluorescence. We counted NK-1 receptor-like immunoreactive cell bodies in laminas I, III-IV, and V-VI of the dorsal horn, ipsilateral to the side of stimulation, from segments L2-L6. NK-1 receptor-like immunoreactivity is uniformly distributed on the surface of cell bodies that do not contain internalized receptors, but in the neurons that have internalized NK-1 receptors, the cytoplasm contains bright, immunofluorescent endosomes. Neurons were considered internalized if they contained $>20$ endosomes in the cell body. All counts are expressed as the percentage of NK-1 receptorimmunoreactive neurons that contain internalized receptor.

Because we found no difference in the magnitude of internalization 
Figure 1. SP and NKA act at the NK-1 receptor at the same concentrations. Left, Intrathecal injection of SP or NKA produces equivalent amounts of NK-1 receptor internalization in the lumbar spinal cord. Percentage of NK-1 receptor-expressing neurons in lamina I of the lumbar spinal cord (L2-L6) showing internalized receptor after intrathecal injection of various doses of SP or NKA ( $n=4-5$ rats). Middle, Incubation of primary spinal cord cultures with SP or NKA results in equivalent NK-1 receptor activation. Percentage of NK-1 receptor-expressing neurons in primary spinal cord cultures showing internalization after incubation for $15 \mathrm{~min}$ in varying concentrations of SP or NKA ( $n=4$ coverslips). Right, Administration of SP or NKA
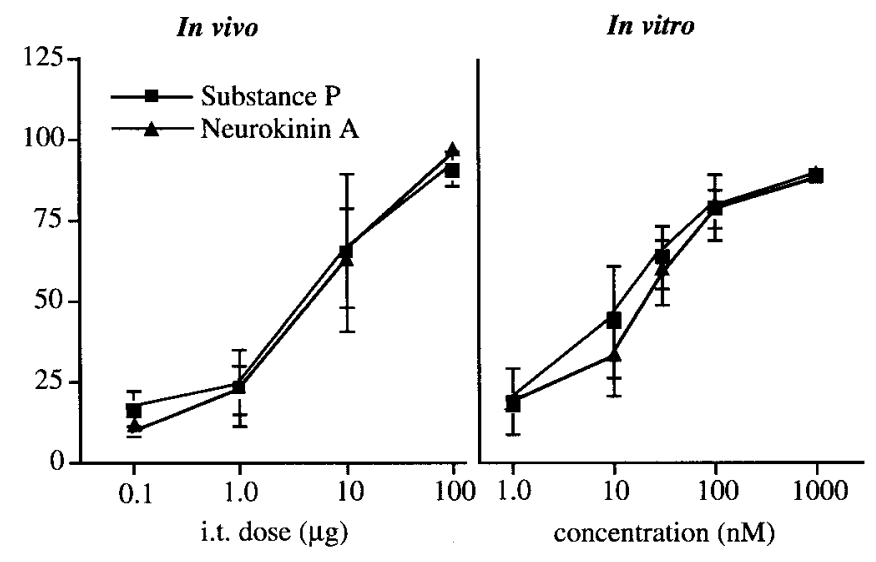

Intracellular calcium

to primary spinal cord cultures produces indistinguishable increases in intracellular calcium. Percentage of the maximal percentage of neurons from primary spinal cord cultures showing an increase of more than two times baseline values after application of varying concentrations of SP or NKA $(n=$ $5-11$ coverslips; maximal percent responders, $\mathrm{SP}=50.66$ and $\mathrm{NKA}=50.38$ ).

along the mediolateral extent of the superficial dorsal horn, we counted all of the neurons within a section, without taking into account the mediolateral position of the cells. Neurons from five sagittal sections were counted from each rat.

Hot plate. To determine whether the NKA-mediated activation of NK-1 receptors contributed to endogenous tachykinin-mediated behaviors, we tested rats treated with GR 205171 in a behavioral paradigm in which mice with a deletion of the PPT-A gene, i.e., lacking SP and NKA, respond differently from their wild-type controls (Cao et al., 1998). PPT-A mutant mice respond normally on a 52.5 or $58.5^{\circ} \mathrm{C}$ hot plate test but show significantly longer response latencies at $55.5^{\circ} \mathrm{C}$. The hot plate test consists of a Plexiglas container with a temperature-controlled floor. The animals are placed on the heated floor and the latency to hindpaw licking or jumping is recorded, at which point the animals are immediately removed. Rats were tested on the hot plate, injected with GR 205171 , and then retested on a $52.5,55.5$, or $58.5^{\circ} \mathrm{C}$ hot plate.

\section{RESULTS}

\section{SP and NKA activate spinal NK-1 receptors at similar concentrations}

To determine whether NKA activates the NK-1 receptor in vivo, we first compared the abilities of exogenously applied NKA and SP to produce NK-1 receptor internalization in the lumbar spinal cord. Increasing doses of NKA and SP were injected intrathecally, and the magnitude of the resulting NK-1 receptor internalization was quantified over the lumbar cord. Both NKA and SP induced NK-1 receptor internalization in the cell bodies and dendrites of lamina I neurons in a dose-dependent manner (Fig. $1 A$ ). We found no difference in the dose-response curves for SP and NKA (two-way ANOVA; $p=0.9538$ ). The $\mathrm{EC}_{50}$ values for NK-1 receptor internalization were $171 \mu \mathrm{M}[95 \%$ confidence interval (C.I.), 66.2-441 $\mu \mathrm{M}$ ] and $210 \mu \mathrm{M}$ (95\% C.I., 87.8-518 $\mu \mathrm{M})$ for SP and NKA, respectively. Finally, neither SP nor NKA induced internalization of NK-1 receptors in lamina III-VI, even at the highest doses tested.

The response of a receptor to an exogenously applied peptide in vivo depends not only on the response of the receptor to the peptide but also on the access of the peptide to the receptor. To be sure that the similar responses seen with NKA and SP in vivo reflected a comparable activity at the NK-1 receptor rather than differences in peptide diff usion or degradation, we next compared the abilities of NKA and SP to induce NK-1 receptor internalization in primary cultures of spinal cord neurons. As in the in vivo experiment, we found that NKA and SP induced NK-1 receptor internalization with an identical dose dependence (Fig. $1 B)$. There was no difference between the number of NK-1 receptor-positive endosomes observed in neurons after incubation with NKA or SP (two-way ANOVA; $p=0.6292$ ). The $\mathrm{EC}_{50}$ values were also identical [SP, $14.28 \mathrm{~nm}$ (95\% C.I., 2.35-87.79 nM); NKA, 26.7 nм (95\% C.I., 1.39-515.00 nM)]. Furthermore, there was no difference in the percentage of neurons that internalized $>10$ endosomes after incubation with NKA or SP [twoway ANOVA; $p=0.9773$; treatment alone, $p=0.6191$; $\mathrm{SP} \mathrm{EC}_{50}$, 15.7 nм (95\% C.I., 13.60-18.17 nм); NKA EC N0 $_{5}, 21.5$ nм (95\% C.I., $14.8-31.2 \mathrm{~nm})]$.

Although NK-1 receptor internalization has been shown to correlate consistently with other indicators of NK-1 receptor activity, receptor internalization is not necessarily a direct marker of receptor-mediated cell signaling. Thus, to specifically address SPand NKA-induced signaling, we also assessed the effects of SP and NKA on intracellular calcium levels in primary spinal cord cultures. The NK-1 receptor signals through Gq/11, activating phospholipase $\mathrm{C}$ and inducing an $\mathrm{IP}_{3}$-mediated release of intracellular calcium stores (Roush and Kwatra, 1998). As we have reported previously for SP, application of NKA to primary spinal cord cultures induced a dose-dependent increase in intracellular calcium concentration in a subpopulation of neurons and glia.

As we found for NK-1 receptor internalization, the doseresponse curves for NKA- and SP-induced calcium increases did not differ (Fig. 1C). We found no difference in the percentage of neurons responding with an increase in intracellular calcium [two-way ANOVA; $p=0.8994$; SP $\mathrm{EC}_{50}, 16.1 \mathrm{~nm}$ (95\% C.I., 4.27-60.5 nм); NKA EC $50,20.53$ nм (95\% C.I., 10.9-37.0 nм)]. This demonstrates that the two peptides not only induce NK-1 receptor internalization at equivalent concentrations but also induce equivalent calcium signaling.

Finally, as we reported previously for SP (Trafton et al., 1999), we found that the dose-response relationships for NKA-induced $\mathrm{NK}-1$ receptor internalization and intracellular calcium increases were identical (data not shown). Thus, NKA-induced signaling occurs at the same doses as receptor internalization, further supporting the use of NK-1 receptor internalization as a marker of tachykinin-mediated activity.

\section{GR 20571 antagonizes NKA- but not SP-induced NK-1 receptor internalization}

We next tested the ability of an NK-1 receptor antagonist, GR 205171, which we reported previously can significantly reduce noxious stimulus-induced NK-1 receptor internalization (Trafton 


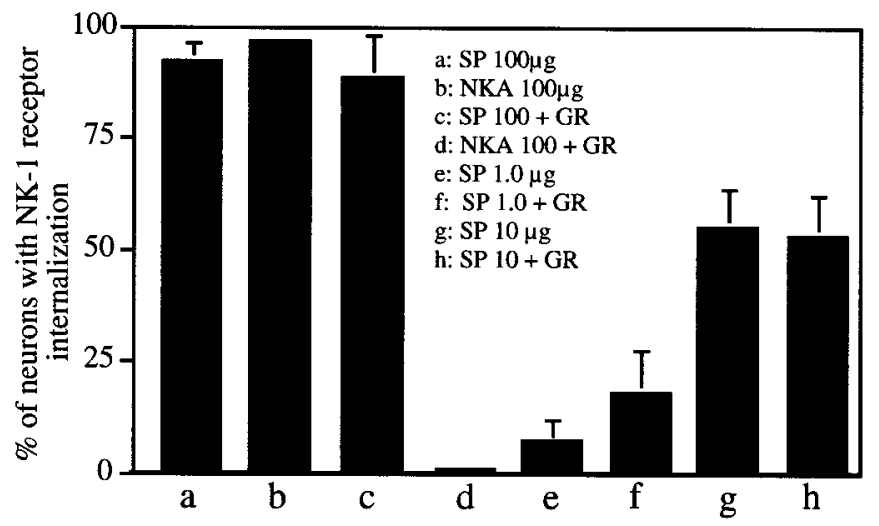

Figure 2. GR205171 prevents NKA- but not SP-induced NK-1 receptor internalization in vivo. Percentage of neurons in lamina I of the lumbar spinal cord (L2-L6) showing internalized NK-1 receptor after intrathecal injection of various doses of SP or NKA in the presence and absence of the NK-1 receptor antagonist $(n=3-6$ rats $)$.

et al., 1999), to prevent the internalization produced by an intrathecal injection of SP or NKA (Fig. 2). We injected rats with 10 $\mathrm{mg} / \mathrm{kg}$ GR 205171 subcutaneously, $20 \mathrm{~min}$ before intrathecal injection of either SP or NKA and then quantified NK-1 receptor internalization in lamina I neurons of the lumbar spinal cord. To our surprise, although GR 205171 completely prevented the NK-1 receptor internalization produced by $100 \mu \mathrm{g}$ of intrathecal NKA $(p=0.0001 ; n=4-6)$, it had no effect on NK-1 receptor internalization induced by intrathecal SP. GR 205171 did not reduce SP-induced NK-1 receptor internalization at any dose (1.0-100 $\mu \mathrm{g} ; 1.0 \mu \mathrm{g}, p=0.6394, n=3-5 ; 10 \mu \mathrm{g}, p=0.8681, n=$ $5 ; 100 \mu \mathrm{g}, p=0.4968, n=5-6)$. Importantly, $1.0-100 \mu \mathrm{g}$ is within the linear range of the SP dose-response curve. Thus, $1.0 \mu \mathrm{g}$ is the lowest dose found to produce NK-1 receptor internalization, and $100 \mu \mathrm{g}$ of SP is a dose that produces maximal internalization (Fig. 3).

To examine this apparent difference in the ability of GR 205171 to block activation of the NK-1 receptor by NKA versus SP, we next looked at the ability of GR 205171 to inhibit NK-1 receptor internalization in primary spinal cord cultures. A great advantage of the in vitro model is that the doses of SP, NKA, and the antagonist are better controlled. We incubated spinal cord cultures in a mixture of $100 \mathrm{~nm} \mathrm{SP}$ or NKA with varying doses of GR 205171 and then quantified NK-1 receptor internalization (Fig. 3). As described above, we found that $100 \mathrm{~nm}$ SP or NKA produced maximal NK-1 receptor internalization. GR 205171 reduced NKA-induced internalization at significantly lower concentrations than were required to inhibit SP-induced NK-1 receptor internalization. Thus, although 10-100 nM GR 205171 significantly inhibited NKA-induced NK-1 receptor internalization, there was no inhibition of SP-induced NK-1 receptor internalization at these concentrations. At higher concentrations, however, GR 205171 did prevent SP-induced NK-1 receptor internalization, demonstrating that, in sufficient excess, the compound is capable of antagonizing SP-mediated NK-1 receptor activation.

\section{GR 205171 reduces noxious stimulus-evoked NK-1 receptor internalization}

Because 10 mg/kg GR 205171 selectively inhibited NKA-induced NK-1 receptor internalization in vivo, we used this dose to estimate the relative contribution of NKA and SP to noxious

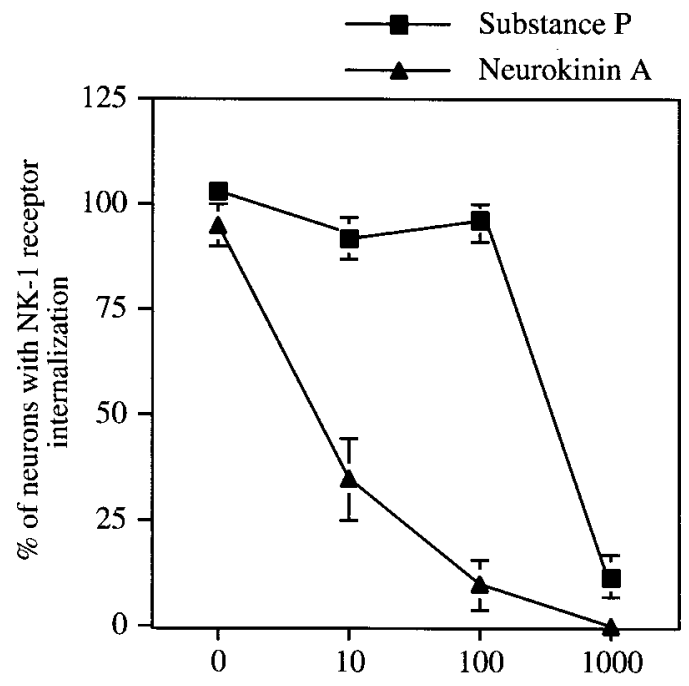

GR $205171(\mathrm{nM})$

Figure 3. GR 205171 selectively inhibits NKA- versus SP-induced NK-1 receptor internalization in vitro. Percentage of NK-1 receptor-expressing neurons in primary spinal cord cultures showing internalization after incubation for $15 \mathrm{~min}$ in varying concentrations of GR 205171 and $100 \mathrm{nM}$ SP or NKA ( $n=4-5$ coverslips).

stimulus-induced NK-1 receptor activation. We have shown previously that noxious thermal, mechanical, or chemical stimulation of the hindpaw induces NK-1 receptor internalization in lamina I neurons of the ipsilateral spinal cord (Abbadie et al., 1997). In the setting of inflammation of the hindpaw, noxious mechanical stimulation produces an even greater magnitude of NK-1 receptor internalization in lamina I neurons of the spinal cord, and neurons in lamina III-VI also contain internalized receptor. In a subsequent report, we showed that $10 \mathrm{mg} / \mathrm{kg}$ GR 205171 reduces $\mathrm{NK}-1$ receptor internalization induced by noxious mechanical stimulation in both normal animals and in animals with an inflammatory injury (Trafton et al., 1999). Here, we reexamined these data in the context of the NKA selectivity of GR 205171 and also assessed the ability of preadministration of $10 \mathrm{mg} / \mathrm{kg} \mathrm{GR}$ 205171 subcutaneously to prevent NK-1 receptor internalization evoked by a noxious thermal stimulus, namely immersion of the hindpaw in $51^{\circ} \mathrm{C}$ water for $2 \mathrm{~min}$.

With all stimuli, GR 205171 significantly reduced NK-1 receptor internalization [thermal, $p<0.0001,45 \%$ fewer neurons showing receptor internalization compared with salinetreated control rats (55\% decrease from saline); mechanical, $p<0.0001,55 \%$ fewer ( $80 \%$ decrease); mechanical with inflammation, $p<0.0001$, lamina I had $45 \%$ fewer $(55 \%$ decrease), lamina III had $24 \%$ fewer ( $94 \%$ decrease), and lamina $\mathrm{V}$ had $8 \%$ fewer $(100 \%$ decrease)]. GR 205171 was less effective in preventing $\mathrm{NK}-1$ receptor internalization in lamina I after noxious mechanical stimulation in the CFA-treated animals (compare SP components in normal rats versus rats with inflammation). However, GR 205171 almost completely prevented NK-1 receptor internalization in lamina III-VI neurons in these same rats.

When we replotted the data to show the relative contributions of NKA (the difference in internalization between saline and GR 205171 groups) and SP (the residual internalization in the GR 205171 group), several trends emerged (Fig. 4). We estimate that 
Thermal

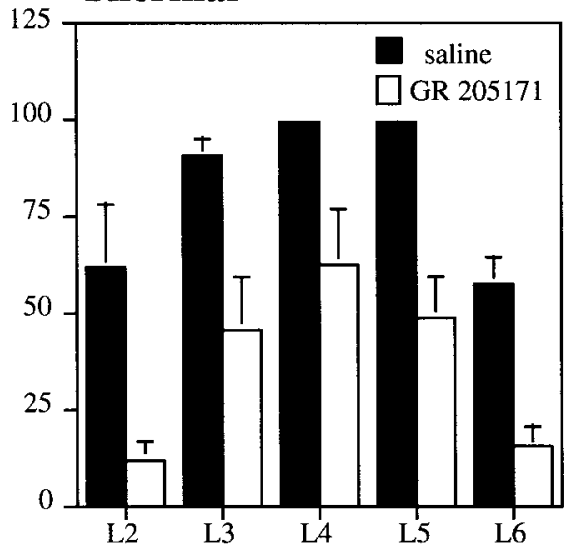

Thermal

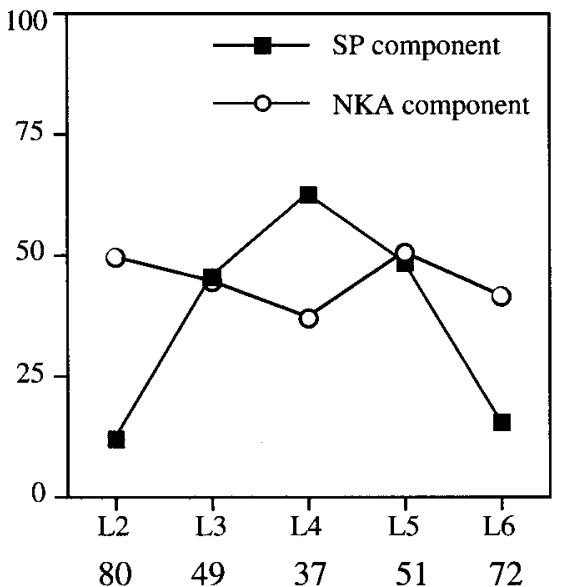

\section{Mechanical}

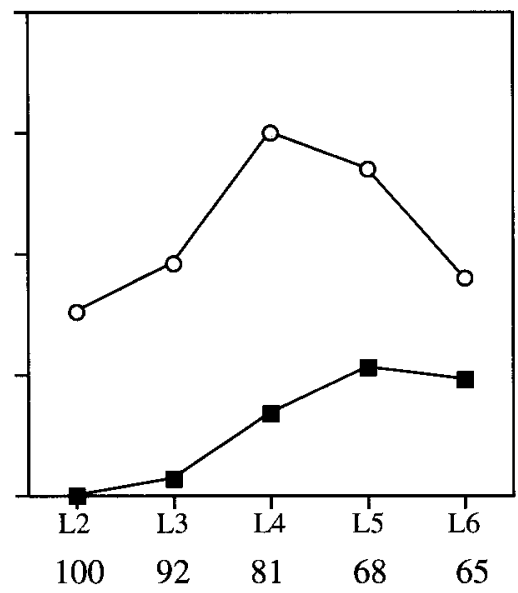

$\%$ NKA contribution

\section{Mechanical with Inflammation}
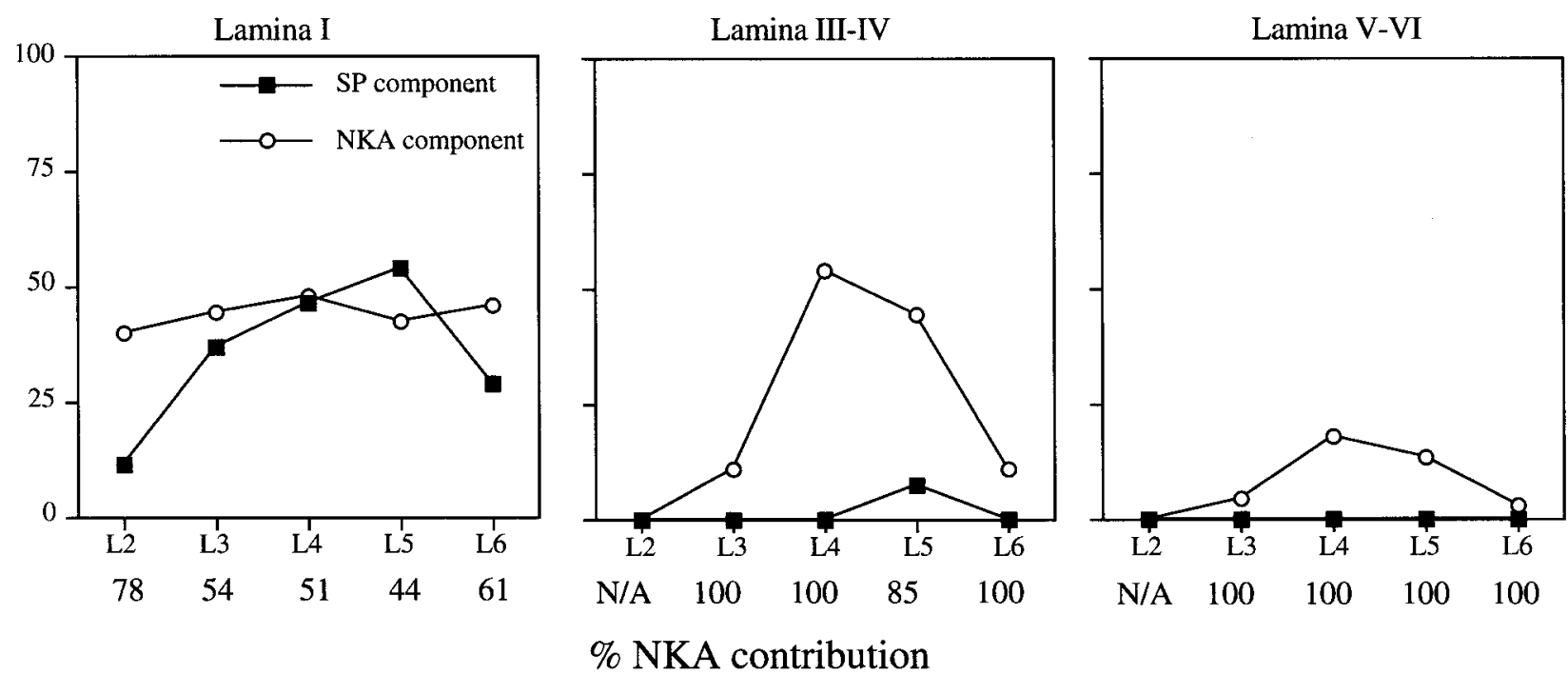

$\%$ NKA contribution

Figure 4. GR 205171 decreases noxious stimulus-induced internalization. Top row, Left, Percentage of NK-1 receptor-expressing neurons showing receptor internalization across the lumbar spinal cord (L2-L6) after a $2 \mathrm{~min}$ immersion of the hindpaw in a $50^{\circ} \mathrm{C}$ water bath in the presence and absence of GR 205171 (10 mg/kg, s.c.; $n=4$ rats). Top row, Middle and Right, Bottom row, Percentage of NK-1 receptor internalization attributable to SP (values in the presence of $10 \mathrm{mg} / \mathrm{kg}$ GR 205171) or NKA (saline values, values with $10 \mathrm{mg} / \mathrm{kg}$ GR 205171) across the lumbar spinal cord (L2-L6) after various noxious stimuli. Top row, Middle, Lamina I neurons after noxious thermal stimulation. Top row, Right, Lamina I neurons after noxious mechanical stimulation. Bottom row, Left, Lamina I neurons after noxious mechanical stimulation in the presence of inflammation. Bottom row, Middle, Lamina III-IV neurons after noxious mechanical stimulation in the presence of inflammation. Bottom row, Right, Lamina V-VI neurons after noxious mechanical stimulation in the presence of inflammation.

the largest SP contribution to internalization in lamina I is seen around L4-L5, the lumbar segments most densely innervated by primary afferents neurons that innervate the hindlimb. In lumbar segments L2, L3, and L6, which receive a much weaker direct primary afferent input from the site of stimulation, we estimate that NKA was responsible for a much larger proportion of the NK-1 receptor internalization. Additionally, we find that NKA is the exclusive contributor to the NK-1 receptor internalization in deeper laminas of the spinal cord (i.e., when the stimulus was applied to the inflamed hindpaw). Of note, however, is the observation that, although NK-1 receptor internalization in the cell bodies of lamina III-V I neurons was completely prevented by GR 205171, we occasionally observed residual NK-1 receptor internalization in the dendrites of these neurons that extend dorsally into lamina I (Fig. 5).
Although we interpret our results as evidence for a direct action of NKA on the NK-1 receptor, the fact that NKA has high affinity for the NK-2 receptor site leaves open the possibility that we are observing an indirect action, through NK-2 receptorexpressing interneurons, or primary afferent terminals that evoke the release of SP. This, of course, cannot account for the in vitro results. To address the question in the in vivo condition, we repeated the study in the presence of intrathecal MEN 11420, a selective NK-2 receptor antagonist. We found that an intrathecal dose of $10 \mu \mathrm{g}$ of MEN-11420 had no effect on NK-1 receptor internalization induced by noxious mechanical stimulation of the hindpaw ( $n=4 ; p=0.8525$; data not shown). Notably, this dose of antagonist did reduce intrathecal NKA-induced defecation when administered identically (data not shown), demonstrating that the dose used was pharmacologically active. 


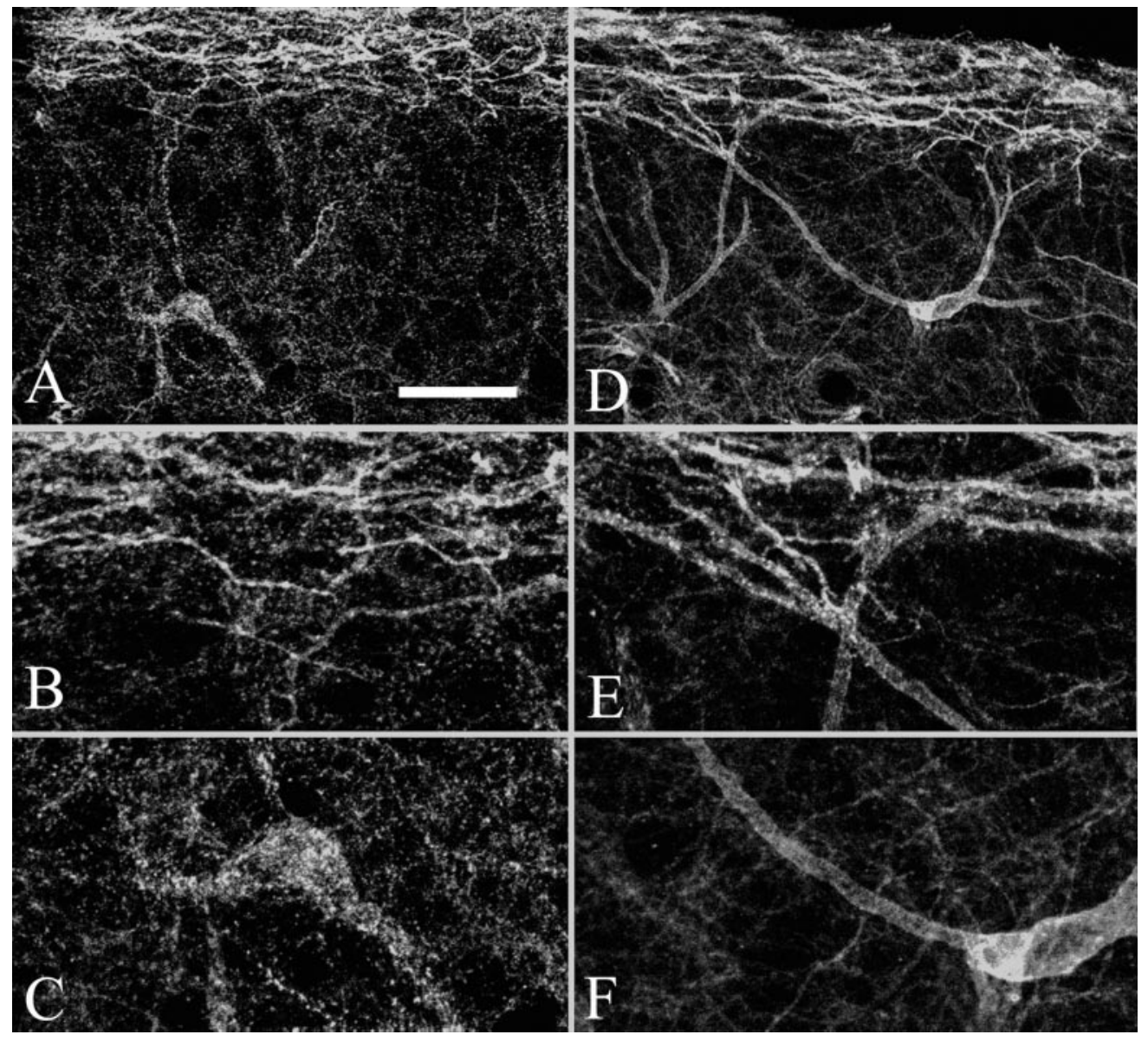

Figure 5. GR 205171 prevents noxious stimulus-evoked NK-1 receptor internalization in neurons ventral to lamina I, which only occurs in the setting of inflammation. NK-1 receptor labeling after noxious mechanical stimulation in the presence of inflammation. $A-C$, With saline. $D-F$, With GR 205171 (10 $\mathrm{mg} / \mathrm{kg}$, s.c.) Note that punctate endosomal labeling ends abruptly at the lamina I-II border in animals treated with GR 2015171. Only the dendrites of cells from deeper lamina that project dorsally to lamina I show internalization $(E)$. The receptor internalization does not extend ventrally toward the cell bodies $(F)$. $B$ and $C$ are higher magnification images of $A . E$ and $F$ are higher magnification images of $D$. Scale bar: $A$, $D, 105 \mu \mathrm{m} ; B, C, E, F, 35 \mu \mathrm{m}$.

\section{GR 205171 has discrete effects on thermal nociception}

Mice lacking the gene for preprotachykinin-A, the precursor protein from which both NKA and SP are derived, show very specific deficits in nociceptive behaviors (Cao et al., 1998). Because these mice lack both SP and NKA, a behavioral analysis in these mice does not provide information on the relative contribution of SP and NKA or of different neurokinin receptors. On the other hand, because GR 205171 only blocks NKA-mediated NK-1 receptor activation, we asked whether administration of this compound could mimic the behavioral phenotype seen in the PPT-A mutant mice, thereby implicating NKA and the NK-1 receptor in these effects.

PPT-A mutant mice and their wild-type controls respond with identical latencies on both a 52.5 and $58.5^{\circ} \mathrm{C}$ hot plate, longer on the former and shorter on the latter. On a $55.5^{\circ} \mathrm{C}$ hot plate, however, there is a striking difference between the mice. The latency of the wild-type mice on the $55^{\circ} \mathrm{C}$ plate is comparable with the $58.5^{\circ} \mathrm{C}$ plates (i.e., it is apparently perceived as hotter than the $52.5^{\circ} \mathrm{C}$ hot plate). In contrast, the PPT-A mutant mice respond at a longer latency on the $55.5^{\circ} \mathrm{C}$ plate, comparable with that seen on the $52.5^{\circ} \mathrm{C}$ hot plate. This suggested that SP and/or NKA contribute to a window of thermal pain sensitivity. In the present study, we tested rats given $10 \mathrm{mg} / \mathrm{kg}$ GR 205171 subcutaneously and their saline controls on the three different hot plate temperatures. Saline-treated rats behaved similarly to the wildtype mice, with a significant decrease in latency to hindpaw licking at the $55.5^{\circ} \mathrm{C}$ temperature. This latency difference was maintained at $58.5^{\circ} \mathrm{C}$. Rats treated with GR 205171 showed an intermediate latency on the $55.5^{\circ} \mathrm{C}$ hot plate, a behavior midway between that seen in the wild-type animals and that seen in the animals that lacked PPT-A gene products (Fig. 6).

\section{DISCUSSION}

\section{Identical effects of SP and NKA}

Our results confirm previous suggestions that SP and NKA have NK-1 receptor-mediated effects at similar concentrations. We found that SP and NKA induced NK-1 receptor internalization and calcium mobilization at the same doses in vivo and in vitro. These results demonstrate that both peptides likely exert their effects via NK-1 receptors.

It is clear, however, that, despite the comparable effects observed, these two tachykinins do not bind the NK-1 receptor identically. Homologous binding studies, in which radiolabeled ligand is displaced by identical, cold ligand, found similar binding affinities for SP and NKA. In contrast, heterologous binding studies, which use different molecules to displace binding, show a much higher affinity of the NK-1 receptor for SP (Hastrup and Schwartz, 1996). Recently, Wijkhuisen et al. (1999) identified a domain near the end of the second extracellular loop of the NK-1 receptor that is necessary for the binding and biological activity of NKA but not SP. This finding suggests that the two neurokinins bind distinct sites on the receptor. It follows that SP and NKA may act on the NK-1 receptor in unique manners. Consistent with this hypothesis, Sagan et al. (1996) found that, whereas $\mathrm{SP}$ increased levels of both cAMP and $\mathrm{IP}_{3}$ in NK-1 receptortransfected Chinese hamster ovary (CHO) cells, NKA only affected $\mathrm{IP}_{3}$. Regardless of their differences in binding characteristics, SP and NKA have been shown to activate aspects of NK-1 
saline

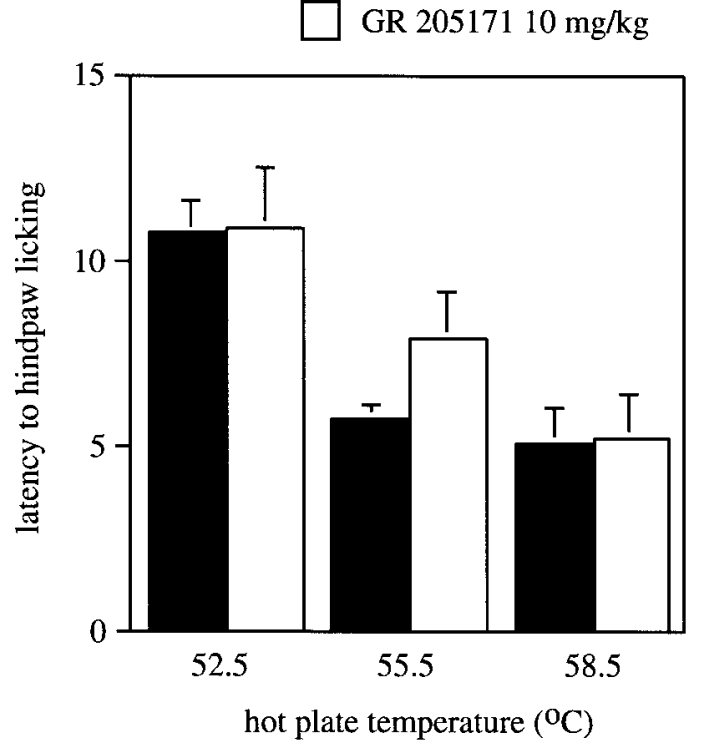

Figure 6. GR 205171 only effects thermal latencies at $55.5^{\circ} \mathrm{C}$, the same stimulus to which PPT-A knock-out mice show increased latencies. Latencies at $55.5^{\circ} \mathrm{C}$ are equivalent to those at $58.5^{\circ} \mathrm{C}$ in saline-treated animals but intermediate to those at 52.5 and $58.5^{\circ} \mathrm{C}$ in animals treated with GR 205171.

receptor signaling at the same doses, demonstrating that they share some functional characteristics (Akasu et al., 1996; Sagan et al., 1996).

\section{Difference in GR 205171 effects on SP- versus NKA-induced activation}

If a receptor has two distinct binding domains, it is theoretically possible to devise compounds that selectively block one site versus the other. We believe that this scenario accounts for the ability of GR 205171 to selectively decrease NKA-mediated NK-1 receptor trafficking. The greatest likelihood is that GR 205171 more directly inhibits the NKA binding portion of the NK-1 receptor. In fact, many other NK-1 receptor antagonists have been shown to have similar properties. For example, CP 96345, FK 888, GR 82,334, RP 67,580, CP-99994, and MEN10581 are better inhibitors of NKA or septide than of SP activation (Maggi, 1995; Jenkinson et al., 1999). For example, using two different NK-1 receptor antagonists, Jenkinson et al. (1999) found that septide-induced NK-1 receptor internalization was inhibited at much lower concentrations than SP-induced NK-1 receptor internalization. The fact that results comparable with ours were found with other NK-1 receptor antagonists reinforces our results and interpretation and suggests that the selectivity of NK-1 receptor antagonists for a subset of NK-1 receptor agonists is not restricted to the antagonist used in this paper.

The selectivity of NK-1 receptor antagonists for NKA versus $\mathrm{SP}$ at this single receptor brings into question the purported selectivity of some of the many NK-1 and NK-2 receptor antagonists. A number of these compounds have been characterized as more or less able to inhibit the actions of SP-like versus NKA-like tachykinins on the assumption that NKA effects were NK-2 receptor-mediated and SP effects were NK-1 receptor-mediated. By this criterion, compounds such as GR 205171, which relatively selectively inhibit NKA-mediated effects through the NK-1 re- ceptor, might be mistakenly characterized as NK-2 receptorselective. Evidence that such mischaracterization has occurred exists. For example, Sagan et al. (1996) used the "NK-2 receptor antagonist" GR 94800 to selectively block NKA-induced activity in NK-1 receptor-transfected CHO cells. Because $\mathrm{CHO}$ cells do not constitutively express neurokinin receptors, this antagonist must also act at the NK-1 receptor. Importantly, the same antagonist had been used previously to argue for the presence of NK-2 receptors in the spinal cord (Lepre et al., 1994). Other studies demonstrating spinal cord NK-2 receptor effects used a different putative NK-2 receptor antagonist, SR 48968, which has been shown to antagonize NK-2 receptors at concentrations orders of magnitude lower than those at which it antagonizes NK-1 receptors. However, the doses chosen for the spinal cord studies were characterized as NK-2 receptor-selective solely on the basis of their selectivity for inhibiting NKA versus SP effects (Neugebauer et al., 1996; Yashpal et al., 1996; Liu and Sandkühler, 1997). As a result, it is possible that the selectivity for NKA versus SP reflected antagonism at an NKA-preferring site on the NK-1 receptor. In light of the new perspective on the properties of the $\mathrm{NK}-1$ receptor, it is clear that great care must be taken when concluding differential actions at NK-1 and NK-2 receptors.

We must emphasize that this paper does not address whether NKA has actions on a spinal cord NK-2 receptor site. We specifically evaluated possible NKA actions at the NK-1 receptor. We can neither confirm nor disprove the hypothesis that NKA exerts additional actions via the NK-2 receptor. Indeed, as noted above, despite the difficulty in identifying and locating NK-2 receptors in the spinal cord, there is pharmacological evidence that such NK-2 receptor-mediated effects occur (FleetwoodWalker et al., 1990, 1993; Munro et al., 1993; Parker et al., 1993; Wiesenfeld-Hallin et al., 1994). Importantly, even if NKA concurrently interacts with $\mathrm{NK}-2$ receptors in the spinal cord, this is unlikely to affect our interpretation of NKA actions that are mediated via the NK-1 receptor. In fact, we found that pharmacologically active doses of an NK-2 receptor antagonist had no effect on noxious stimulus-evoked NK-1 receptor internalization, demonstrating that NK-2 receptor activation is not involved in the process of spinal NK-1 receptor activation.

Although we clearly demonstrated a difference in the antagonism by GR 205171 of NKA versus SP effects, our in vivo experiments assume that the dose of GR 205171 that we used only inhibits NKA-mediated NK-1 receptor activation. Our data supports this assumption. GR $205171(10 \mathrm{mg} / \mathrm{kg})$ did not reduce NK-1 receptor internalization produced by a dose of SP that induces $<25 \%$ of maximal internalization. Because GR 205171 did not prevent even threshold levels of NK-1 receptor internalization, our results cannot be explained by arguing that the SP concentration was supramaximal and, therefore, not blockable. Note that we have shown previously that $1.0 \mathrm{mg} / \mathrm{kg}$ GR 205171 does not reduce noxious stimulus-induced NK-1 receptor internalization and that $10 \mathrm{mg} / \mathrm{kg}$ only produces a partial reduction (Trafton et al., 1999). Thus, the dose of antagonist used here is at most 10 times greater than an ineffective dose and therefore in a range in which strictly NKA-selective effects would be expected.

\section{Magnitude of NKA-induced NK-1 receptor activity}

We demonstrate that GR 205171, at doses that do not block the action of SP, can primarily prevent noxious stimulus-induced NK-1 receptor internalization. This suggests that, under normal physiological conditions, NKA mediates a significant proportion of NK-1 receptor activation and signaling. Despite the evidence 
that SP and NKA activate the NK-1 receptor at similar doses, given the well documented ability of SP to displace NKA from binding to the receptor in heterologous binding studies, this result is very surprising (Hastrup and Schwartz, 1996). Because the peptides are coreleased in vivo, one might expect that SP would be the predominant NK-1 receptor agonist, simply because of its higher affinity for the NK-1 receptor. There are, however, numerous possible explanations for the greater effect of NKA on dorsal horn NK-1 receptors. For example, Bakhle and Bell (1995) observed that SP and NKA were not evenly distributed throughout sensory neurons. They found a greater proportion of SP expressed in the peripheral terminals and dorsal root ganglion and a greater proportion of NKA in the spinal cord terminals. Thus, it is possible that primary afferent fibers release more NKA than SP into the dorsal horn during noxious stimulation. Furthermore, it has been shown that NKA is less susceptible to degradation by endogenous endopeptidases (Nyberg et al., 1984; Theodorsson-Norheim et al., 1987) Thus, SP may be degraded too rapidly to have full effects, leaving ample receptor on which the less degradable NKA could act.

This difference in peptide degradation may also contribute to the preferential activation of NK-1 receptors by NKA in deeper laminas of the spinal cord during inflammatory injury. We found that nearly all of the NK-1 receptor internalization seen in lamina III-V I could be blocked by GR 205171. We conclude, therefore, that NK-1 receptor signaling in deeper laminas is primarily triggered by NKA. This suggestion is supported by the results of Hope et al. (1990) who demonstrated that NKA can be detected more ventrally in the spinal cord gray matter than SP after joint stimulation in the setting of peripheral inflammation.

\section{Functional relevance to signal transduction and behavior}

Although SP and NKA appear to have similar effects on most aspects of NK-1 receptor signaling (electrophysiological, calcium- $\mathrm{IP}_{3}$, and internalization), qualitative differences in SP and NKA signaling through the NK-1 receptor have been reported. Of note, SP, but not NKA, increases cAMP levels in CHO cells that express the NK-1 receptor (Sagan et al., 1996). This suggests that SP and NKA may exert different effects on target neurons through the same receptor.

Because the two peptides are differentially degraded, this could allow for different signaling through the NK-1 receptor in different target regions. Microprobe studies have shown that NKA is found more evenly throughout the dorsal horn after noxious stimulation than is SP (Duggan et al., 1990). Our data are consistent with this result. We found that the NK-1 receptor internalization induced by NKA was distributed over a greater rostrocaudal area than that produced by SP. Based on the residual internalization observed in the presence of GR 205171, we reasoned that the SP contribution is focused at the site of stimulated primary afferent input, namely the L4-L5 segments. It follows that the neurons closest to the site of release would be preferentially activated by SP, whereas those more distant from the release site would be activated by NKA. This might not only produce different regional patterns of signaling in the spinal cord, but there may also be differences in NK-1 receptor signaling in different domains of individual neurons, depending on their proximity to synaptic terminals that release the tachykinins. As shown in Figure 5, this is most easily demonstrated in the case of deeper dorsal horn neurons, but it is also likely that this occurs in lamina I. This latter possibility, however, is difficult to assess, because the dendritic trees of lamina I neurons overlap extensively and are thus difficult to trace back to their cell bodies. Analysis of the responses of the NK-1 receptor neuron population (Fig. 4) with GR 205171 also suggests that this may be occurring.

The functional relevance of differential activation of $\mathrm{NK}-1$ receptors by SP versus NKA is not clear. However, given the differences in signal transduction pathways stimulated, it is not unreasonable to speculate that the two peptides have qualitatively different effects on signaling and gene transcription in the same neurons.

In behavioral studies, we found that rats given GR 205171 showed thermal nociceptive responsiveness midway between that seen in PPT null mice and their wild-type controls. This suggests that both SP and NKA contribute to nociceptive behaviors via the NK-1 receptor and that SP cannot produce wild-type behavior in the absence of NKA. Future studies that more extensively examine the behavioral effects of GR 205171, including changes in inflammation, different noxious stimulus modalities, spreadextent of hyperalgesia, and receptive field changes, will more precisely distinguish the differential contribution of SP and NKA at the NK-1 receptor.

\section{REFERENCES}

Abbadie C, Trafton JA, Liu H, Mantyh PW, Basbaum AI (1997) Inflammation increases the distribution of dorsal horn neurons the internalize the neurokinin-1 receptor in response to noxious and non-noxious stimulation. J Neurosci 17:8049-8060.

Akasu T, Ishimatsu M, Yamada K (1996) Tachykinins cause inward current through NK-1 receptors in bullfrog sensory neurons. Brain Res 713:160-167.

Bakhle YS, Bell C (1995) Neurokinin A and substance P vary independently in different regions of rat sensory neurons. Neuropeptides 28:237-241.

Cao Y, Mantyh PM, Carlson EJ, Gillespie AM, Epstein CJ, Basbaum AI (1998) Primary afferent tachykinins are required to experience moderate to intense pain. Nature 392:390-394.

De Koninck Y, Henry JL (1991) Substance P-mediated slow excitatory postsynaptic potential elicited in dorsal horn neurons in vivo by noxious stimulation. Proc Natl Acad Sci USA 88:11344-11348.

Duggan AW, Hope PJ, Jarrott B, Schaible HG, Fleetwood-Walker S (1990) Release, spread and persistence of immunoreactive neurokinin $\mathrm{A}$ in the dorsal horn of the cat following noxious cutaneous stimulation. Studies with antibody microprobes. Neuroscience 35:195-202.

Fleetwood-Walker SM, Mitchell R, Hope PJ, El-Yassir N, Molony V, Bladon CM (1990) The involvement of neurokinin receptor subtypes in somatosensory processing in the superficial dorsal horn of the cat. Brain Res 519:169-182.

Fleetwood-Walker SM, Parker RMC, Munro FE, Young MR, Hope PJ, Mitchell R (1993) Evidence for a role of tachykinin NK2 receptors in mediating brief nociceptive inputs to rat dorsal horn (laminae III-V) neurons. Eur J Pharmacol 242:173-181.

Gamse R, Saria A (1986) Nociceptive behavior after intrathecal injections of substance $\mathrm{P}$, neurokinin $\mathrm{A}$ and calcitonin gene-related peptide in mice. Neurosci Lett 70:143-147.

Hastrup H, Schwartz TW (1996) Septide and neurokinin A are highaffinity ligands of the NK-1 receptor: evidence from homologous versus heterologous binding analysis. FEBS Lett 399:264-266.

Henry JL (1976) Effects of substance P on functionally identified units in cat spinal cord. Brain Res 114:439-451.

Hope PJ, Jarrot B, Schaible HG, Clarke RW, Duggan AW (1990) Release and spread of immunoreactive neurokinin A in the cat spinal cord in a model of acute arthritis. Brain Res 533:292-299.

Hylden JL, Wilcox GL (1981) Intrathecal substance P elicits a caudallydirected biting and scratching behavior in mice. Brain Res 217:212-215.

Jenkinson KM, Southwell BR, Furness JB (1999) Two affinities for a single antagonist at the neuronal NK1 tachykinin receptor: evidence from quantitation of receptor endocytosis. $\mathrm{Br} \mathrm{J}$ Pharmacol 126:131-136.

Lepre M, Olpe HR, Evans RH, Brugger F (1994) Physiological and pharmacological characterization of the spinal tachykinin NK-2 receptor. Eur J Pharmacol 258:23-31.

Liu XG, Sandkühler J (1997) Characterization of long-term potentiation of c-fiber-evoked potentials in spinal dorsal horn of adult rat: essential role of NK-1 and NK-2 receptors. J Neurophysiol 78:1973-1982.

Maggi CA (1995) The mammalian tachykinin receptors. Gen Pharmacol 26:911-944. 
Munro FE, Fleetwood-Walker SM, Parker RMC, Mitchell R (1993) The effects of neurokinin receptor antagonists on mustard oil-evoked activation of rat dorsal horn neurons. Neuropeptides 25:299-305.

Neugebauer V, Rumenapp P, Schaible HG (1996) The role of spinal neurokinin-2 receptors in the processing of nociceptive information from the joint and in the generation and maintenance of inflammationevoked hyperexcitability of dorsal horn neurons in the rat. Eur J Neurosci 8:249-260.

Nyberg F, Le Greves P, Sundqvist C, Terenius L (1984) Characterization of substance $\mathrm{P}(1-7)$ and (1-8) generating enzyme in human CSF. Biochem Biophys Res Commun 125:244-250.

Parker RM, Fleetwood-Walker SM, Rosie R, Munro FE, Mitchell R (1993) Inhibition by NK2 but not NK1 antagonists of carrageenaninduced preprodynorphin mRNA expression in rat dorsal horn lamina I neurons. Neuropeptides 25:213-222.

Radhakrishnan V, Henry JL (1997) Electrophysiological evidence that neurokinin A acts via NK-1 receptors in the cat dorsal horn. Eur J Neurosci 9:1977-1985.

Roush ED, Kwatra MM (1998) Human substance P receptor expressed in Chinese hamster ovary cells directly activates Gaq/11, Gas and Gao. FEBS Lett 428:291-294.

Sagan S, Chassaing G, Pradier L, Laville S (1996) Tachykinin peptides affect differently the second messenger pathways after binding to $\mathrm{CHO}$ expressed human NK-1 receptors. J Pharmacol Exp Ther 276:1039-1048.

Theodorsson-Norheim E, Hemsen A, Brodin E, Lundberg JM (1987)
Sample handling techniques when analyzing regulatory peptides. Life Sci 41:845-848.

Trafton JA, Abbadie C, Marchand S, Mantyh PW, Basbaum AI (1999) Spinal opioid analgesia: how critical is the regulation of substance $\mathrm{P}$ signaling? J Neurosci 19:9642-9653.

Vigna SR, Bowden JJ, McDonald DM, Fisher J, Okamoto A, McVey DC, Payan DG, Bunnett NW (1994) Characterization of antibodies to the rat substance $\mathrm{P}(\mathrm{NK}-1)$ receptor and to a chimeric substance $\mathrm{P}$ receptor expressed in mammalian cells. J Neurosci 14:834-845.

Wiesenfeld-Hallin Z, Luo L, Xu XJ, Maggi CA (1994) Differential effects of selective tachykinin NK2 receptor antagonists in rat spinal cord. Eur J Pharmacol 251:99-102.

Wijkhuisen A, Sagot MA, Frobert Y, Creminon C, Grassi J, Boquet D, Couraud JY (1999) Identification in the NK-1 tachykinin receptor of a domain involved in recognition of neurokinin $\mathrm{A}$ and septide but not of substance P. FEBS Lett 447:155-159.

Yashpal K, Hui-Chan CWY, Henry JL (1996) SR 48968 specifically depresses neurokinin A- vs substance P-induced hyperalgesia in a nociceptive withdrawal reflex. Eur J Pharmacol 308:41-48.

Yu A, Hertz E, Hertz L (1984) Alterations in uptake and release rates for GABA, glutamate, and glutamine during biochemical maturation of highly purified cultures of cerebral cortical neurons, a GABAergic preparation. J Neurochem 42:951-959.

Zerari F, Karpitskiy V, Krause J, Descarries L, Couture R (1998) Astroglial distribution of neurokinin-2 receptor immunoreactivity in the rat spinal cord. Neuroscience 84:1233-1246. 